\title{
Regularity criteria for the 3D magneto-micropolar fluid equations in the Morrey-Campanato space
}

\author{
Sadek Gala
}

\begin{abstract}
In this paper, some improved regularity criteria for the 3D magneto-micropolar fluid equations are established in Morrey-Campanato spaces. It is proved that if the velocity field satisfies

$u \in L^{\frac{2}{1-r}}\left(0, T ; \dot{\mathcal{M}}_{p, \frac{3}{r}}\left(\mathbb{R}^{3}\right)\right) \quad$ with $r \in(0,1)$ or $u \in C\left(0, T ; \dot{\mathcal{M}}_{p, \frac{3}{r}}\left(\mathbb{R}^{3}\right)\right)$

or the gradient field of velocity satisfies

$$
\nabla u \in L^{\frac{2}{2-r}}\left(0, T ; \dot{\mathcal{M}}_{p, \frac{3}{r}}\left(\mathbb{R}^{3}\right)\right) \text { with } r \in(0,2),
$$
\end{abstract}

then the solution remains smooth on $[0, T]$. By the embedding $L^{\frac{3}{r}} \subset$ $\dot{\mathcal{M}}_{p, \frac{3}{r}}$, we see that our result is an improvement of (Yuan in Acta Mathematica Scientia, to appear).

Mathematics Subject Classification (2000). 35Q35, 76W05, 35B65.

Keywords. Magneto micropolar fluid equations, Regularity criterion, Morrey-Campanato spaces.

\section{Introduction}

This paper concerns about the regularity of weak solutions and blow-up criteria of smooth solutions to the magneto-micropolar fluid equations in three dimensions:

$$
\left\{\begin{array}{l}
\partial_{t} u+(u \cdot \nabla) u-(\mu+\chi) \Delta u-b \cdot \nabla b+\nabla\left(p+b^{2}\right)-\chi \nabla \times \omega=0, \\
\partial_{t} \omega-\gamma \Delta \omega-\kappa \nabla \operatorname{div} \omega+2 \kappa \omega+u \cdot \nabla \omega-\chi \nabla \times u=0 \\
\partial_{t} b-\nu \Delta b+u \cdot \nabla b-b \cdot \nabla u=0 \\
\nabla \cdot u=\nabla \cdot b=0 \\
u(x, 0)=u_{0}(x), b(x, 0)=b_{0}(x), \omega(x, 0)=\omega_{0}(x),
\end{array}\right.
$$


where $u=\left(u_{1}(x, t), u_{2}(x, t), u_{3}(x, t)\right)$ denotes the velocity of The fluids at a point $x \in \mathbb{R}^{3}, t \in[0, T), \omega=\left(\omega_{1}(x, t), \omega_{2}(x, t), \omega_{3}(x, t)\right), b=\left(b_{1}(x, t), b_{2}(x, t)\right.$, $\left.b_{3}(x, t)\right)$ and $p=p(x, t)$ denote, respectively, the micro-rotational velocity, the magnetic field and the hydrostatic pressure. $u_{0}, \omega_{0}$ and $b_{0}$ are the prescribed initial data for the velocity and angular velocity and magnetic field with properties $\operatorname{div} u_{0}=0$ and $\operatorname{div} b_{0}=0 . \mu$ is the kinematic viscosity, $\chi$ is the vortex viscosity, $\kappa$ and $\gamma$ are spin viscosities, and $\frac{1}{\nu}$ is the magnetic Reynold. If the magnetic field $b=0,(1.1)$ reduces to the micropolar fluid system. Theory of micropolar fluids was first proposed by Eringen [4] in 1966, which enables us to consider some physical phenomena that cannot be treated by the classical Navier-Stokes equations for the viscous incompressible fluids, for example, the motion of animal blood, liquid crystals and dilute aqueous polymer solutions etc. The existences of weak and strong solutions were treated by Galdi and Rionero [7], and Yamaguchi [23], respectively. If, further, the vortex viscosity $\chi=0$, the velocity $u$ does not depend on the micro-rotation field $\omega$, and the first equation reduces to the classical Navier-Stokes equation which has been greatly analyzed, see, for example, the classical books by Ladyzhenskaya [13], Lions [15] or Lemarié-Rieusset [14]. If we ignore the micro-rotation of particles, it reduces to the viscous incompressible magnetohydrodynamic equations, which has also been studied extensively [1-3,9,20]. It is worthy to note that He and Xin [9], Zhou [25,26] proved the regularity criteria of weak solutions to the magneto-hydrodynamic equations, which only need the velocity $u$ or its gradient $\nabla u$ or the vorticity $\nabla \times u$ or $p$ and $b$ or $\nabla p$ and $b$ to satisfy some conditions. Later, He and Wang [8] extended the results by Marcinkiewcz spaces, see [9]. For the generalized viscous MHD equations, Zhou [27], recently, also obtained some results on regularity criteria. These all indicate that the velocity field $u$ plays a more dominate role than the magnetic field $b$ does on the regularity of solutions to the magneto-hydrodynamic equations.

The magneto-micropolar fluid system (1.1) was studied by Galdi and Rionero in [7]. Rojas-Medar [18] studied it and established the local in time existence and uniqueness of strong solutions by the spectral Galerkin method. Ortega-Torres and Rojas-Medar [17] proved global in time existence of strong solution for small initial data. Rojas-Medar and Boldrini [19] proved the existence of weak solutions by the Galerkin method, and in 2D case, also proved the uniqueness of the weak solutions. Recently, Ferreira and Villamizar-Roa [5] considered the existence and stability of solutions to the micropolar fluids in exterior domains. Villamizar-Roa and Rodríguez-Bellido [22] studied the micropolar system in a bounded domain using the semigroup approach in $L^{p}$, showing the global existence of strong solutions for small data and the asymptotic behavior and stability of the solutions.

The regularity criteria of weak solutions to the system (1.1) play an important role to understanding the physical essence of the micropolar fluid motion. As it is demonstrated in reference $[8,9]$, we also prove that to secure the regularity of weak solutions to (1.1), one only needs to impose conditions on the velocity field of the fluids. This also demonstrates that in the regularity 
of weak solutions the micro-rotational velocity $\omega$ of particles and the magnetic field $b$ play a less important role than the velocity $u$ does, and the regularity of weak solutions to (1.1) is dominated by the velocity $u$ of the fluids.

Recently, regularity criteria were established in [24] on the velocity field in terms of $L^{p}$ space. In order to understand the regular solutions of the magneto-micropolar fluid equations from the point of view of homogeneity, we will consider problem (1.1) in homogeneous spaces. The typical homogeneous spaces are the Lebesgue spaces $L^{p}\left(0, T ; L^{q}\left(\mathbb{R}^{3}\right)\right)$ and $L^{p}\left(\mathbb{R}^{3}\right)$ whose degree is $-\left(\frac{3}{p}+\frac{2}{q}\right)$ (as done in $\left.[24]\right)$.

The purpose of this paper is to improve and extend some known regularity criterion of weak solution for the magneto-micropolar fluid equations in the Morrey-Campanato space $\dot{\mathcal{M}}_{p, \frac{3}{r}}$ (see Definition in Sect. 2). It is a natural way to extend the space widely and improve the previous results.

\section{Morrey-Campanato spaces}

We recall the definition and some properties of the space that we are going to use. These spaces play an important role in studying the regularity of solutions to partial differential equations (see e.g. $[12,14]$ and references therein).

Definition 2.1. For $1<p \leq q \leq+\infty$, the Morrey-Campanato space $\dot{\mathcal{M}}_{p, q}$ is defined by:

$$
\dot{\mathcal{M}}_{p, q}=\left\{f \in L_{l o c}^{p}\left(\mathbb{R}^{3}\right):\|f\|_{\dot{\mathcal{M}}_{p, q}}=\sup _{x \in \mathbb{R}^{3}} \sup _{R>0} R^{3 / q-3 / p}\|f\|_{L^{p}(B(x, R)}<\infty\right\},
$$

where $B(x, R)$ denotes the closed ball in $\mathbb{R}^{3}$ with center $x$ and radius $R$.

It is easy to check the following:

$$
\|f(\lambda .)\|_{\dot{\mathcal{M}}_{p, q}}=\frac{1}{\lambda^{\frac{3}{q}}}\|f\|_{\dot{\mathcal{M}}_{p, q}}, \quad \lambda>0 .
$$

Hence, for any $f(x, t)$ defined for both spatial and time variables,

$$
\left\|f_{\lambda}\right\|_{L^{\frac{2}{1-r}}}\left((0, T) ; \dot{\mathcal{M}}^{2, \frac{3}{r}}\left(\mathbb{R}^{3}\right)\right)=\|f\|_{L^{\frac{2}{1-r}}\left((0, T) ; \dot{\mathcal{M}}^{2, \frac{3}{r}}\left(\mathbb{R}^{3}\right)\right)},
$$

for any $\lambda>0$ with $f_{\lambda}(x, t)=\lambda f\left(\lambda x, \lambda^{2} t\right)$. Here, the point is that if $(u, \omega, b)$ solves the magneo-micropolar fluid equations, then so does $\left(u_{\lambda}, \omega_{\lambda}, b_{\lambda}\right)$ for all $\lambda>0$. This is so called scaling dimension zero property.

We shall assume the following classical results [10,21]:

a. For $1 \leq p \leq p^{\prime}, p \leq q \leq+\infty$ and for all function $f$ so that $f \in \mathcal{M}_{p, q} \cap L^{\infty}$ :

$$
\|f\|_{\ddot{\mathcal{M}}_{p^{\prime}, q \frac{p^{\prime}}{p}}} \leq\|f\|_{L^{\infty}}^{1-\frac{p}{p^{\prime}}}\|f\|_{\dot{\mathcal{M}}_{p, q}}^{\frac{p}{p^{\prime}}} .
$$


b. For $p, q, p^{\prime}, q^{\prime}$ so that $\frac{1}{p}+\frac{1}{p^{\prime}} \leq 1, \frac{1}{q}+\frac{1}{q^{\prime}} \leq 1, f \in \dot{\mathcal{M}}_{p, q}, g \in \dot{\mathcal{M}}_{p^{\prime}, q^{\prime}}$. Then

$$
f g \in \mathcal{M}_{p^{\prime \prime}, q^{\prime \prime}} \quad \text { with } \frac{1}{p}+\frac{1}{p^{\prime}}=\frac{1}{p^{\prime \prime}}, \quad \frac{1}{q}+\frac{1}{q^{\prime}}=\frac{1}{q^{\prime \prime}} .
$$

c. For $1 \leq p \leq 3$, we have

$$
\forall \lambda>0, \quad\|\lambda f(\lambda .)\|_{\dot{\mathcal{M}}_{p, 3}}=\|f\|_{\dot{\mathcal{M}}_{p, 3}} .
$$

d. For $1 \leq p \leq q<\infty$, we have the inclusion relation

$$
L^{q}=\dot{\mathcal{M}}_{q, q} \subset \dot{\mathcal{M}}_{p, q}
$$

Additionally, for $2<p \leq \frac{3}{r}$ and $0 \leq r<\frac{3}{2}$, we have the following inclusion relations:

$$
L^{\frac{3}{r}}\left(\mathbb{R}^{3}\right) \subset L^{\frac{3}{r}, \infty}\left(\mathbb{R}^{3}\right) \subset \dot{\mathcal{M}}_{p, \frac{3}{r}}\left(\mathbb{R}^{3}\right),
$$

where $L^{p, \infty}$ denotes the usual Lorentz (weak $L^{p}$ ) space. The first inclusion is well known. The second relation

$$
L^{\frac{3}{r}, \infty}\left(\mathbb{R}^{3}\right) \subset \dot{\mathcal{M}}_{p, \frac{3}{r}}\left(\mathbb{R}^{3}\right)
$$

is shown as follows.

$$
\begin{aligned}
\|f\|_{\mathcal{M}_{p, \frac{3}{r}}} & \leq \sup _{E}|E|^{\frac{r}{3}-\frac{1}{p}}\left(\int_{E}|f(y)|^{p} d y\right)^{\frac{1}{p}} \quad\left(f \in L^{\frac{3}{r}, \infty}\left(\mathbb{R}^{3}\right)\right) \\
& =\left(\sup _{E}|E|^{\frac{p r}{3}-1} \int_{E}|f(y)|^{p} d y\right)^{\frac{1}{p}} \\
& \cong\left(\sup _{R>0} R\left|\left\{x \in \mathbb{R}^{3}:|f(y)|^{p}>R\right\}\right|^{\frac{p r}{3}}\right)^{\frac{1}{p}} \\
& =\sup _{R>0} R\left|\left\{x \in \mathbb{R}^{3}:|f(y)|>R\right\}\right|^{\frac{r}{3}} \\
& \cong\|f\|_{L^{\frac{3}{r}, \infty}} .
\end{aligned}
$$

Since this space $\dot{\mathcal{M}}_{p, \frac{3}{r}}$ is wider than $L^{\frac{3}{r}}$, the above regularity criterion is an improvement of Yuan's result and hence our regularity criterion covers the recent result given by Yuan [24].

Definition 2.2 (The predual $\mathcal{Z}^{p^{\prime}, q^{\prime}}[\mathbf{1 4}]$ ). Let $1 \leq q^{\prime} \leq p^{\prime}<\infty$. We define the following homogeneous space $\mathcal{Z}^{p^{\prime}, q^{\prime}}$ as the subspace of $L^{q^{\prime}}\left(\mathbb{R}^{3}\right)$ of functions $f$, which can be decomposed into an atomic series

$$
f=\sum_{k \in \mathbb{N}} g_{k}
$$

where the functions $\left(g_{k}\right)_{k} \subset L_{\text {comp }}^{p^{\prime}}\left(\mathbb{R}^{3}\right)$ and satisfy the following inequalities for the diameter $d_{k}$ of the support of $g_{k}: d_{k} \leq 1$ and

$$
\sum_{k \in \mathbb{N}} d_{k}^{3\left(\frac{1}{q^{\prime}}-\frac{1}{p^{\prime}}\right)}\left\|g_{k}\right\|_{L^{p^{\prime}}}<\infty .
$$


$\mathcal{Z}^{p^{\prime}, q^{\prime}}$ is a Banach space when it is equipped with the norm:

$$
\|f\|_{\mathcal{Z}^{p^{\prime}, q^{\prime}}}=\inf \left\{\sum_{k \in \mathbb{N}} d_{k}^{3\left(\frac{1}{q^{\prime}}-\frac{1}{p^{\prime}}\right)}\left\|g_{k}\right\|_{L^{p^{\prime}}} \quad \text { such that } f=\sum_{k \in \mathbb{N}} g_{k}\right\},
$$

where the infimum is taken over all possible decompositions of $f$ into an atomic series.

Notice that

$$
L_{\text {comp }}^{p^{\prime}} \hookrightarrow \mathcal{Z}^{p^{\prime}, q^{\prime}} \hookrightarrow L^{q^{\prime}, 1} .
$$

We begin with the following lemma which is essentially due to Lemarié -Rieusset [14].

Lemma 2.1. For $q^{\prime}<p^{\prime}<p^{\prime \prime}$, we have

$$
\mathcal{Z}^{p^{\prime \prime}, q^{\prime}} \subset \mathcal{Z}^{p^{\prime}, q^{\prime}} .
$$

Proof. This is a well-known consequence of Hölder and Minkowski inequalities and the following one

$$
\left\|g_{k}\right\|_{L^{p^{\prime}}} \leq\left(C d_{k}^{3}\right)^{\left(\frac{1}{p^{\prime}}-\frac{1}{p^{\prime \prime}}\right)}\left\|g_{k}\right\|_{L^{p^{\prime \prime}}} .
$$

For the proof of Theorems 3.1 and 3.2, we state the following lemma.

Lemma 2.2. Let $1 \leq q^{\prime} \leq p^{\prime}<\infty$. Then if we take $p$ and $q$ such that $\frac{1}{p^{\prime}}+\frac{1}{p}=1$, $\frac{1}{q}+\frac{1}{q^{\prime}}=1$, we have

$$
\left(\dot{\mathcal{M}}^{p, q}\right)^{*}=\mathcal{Z}^{p^{\prime}, q^{\prime}}
$$

Proof. The result is due to [14]; but we give here a detailed proof for the reader's convenience. We first remark that as $L_{\text {comp }}^{p^{\prime}} \subset \mathcal{Z}^{p^{\prime}, q^{\prime}}$ and thus $\left(\mathcal{Z}^{p^{\prime}, q^{\prime}}\right)^{*} \subset L_{\text {loc }}^{p}$. Assume $f \in \mathcal{Z}^{p^{\prime}, q^{\prime}}$ and take $h \in \dot{\mathcal{M}}^{p, q}$, then we have for all decomposition

$$
\begin{aligned}
f \stackrel{L^{p^{\prime}}}{=} \sum_{k \in \mathbb{N}} g_{k} & \\
\left|\int_{\mathbb{R}^{3}} f h d x\right| & \leq \sum_{k \in \mathbb{N}} \int_{B\left(x_{k}, d_{k}\right)}\left|g_{k} h\right| d x \\
& \leq \sum_{k \in \mathbb{N}} d_{k}^{3\left(\frac{1}{p}-\frac{1}{q}\right)}\left\|g_{k}\right\|_{L^{p^{\prime}}} d_{k}^{3\left(\frac{1}{q}-\frac{1}{p}\right)}\left(\int_{B\left(x_{k}, d_{k}\right)}|h|^{p} d x\right)^{\frac{1}{p}} \\
& \leq\|h\|_{\mathcal{M}^{p, q}} \sum_{k \in \mathbb{N}} d_{k}^{3\left(\frac{1}{q^{\prime}}-\frac{1}{p^{\prime}}\right)}\left\|g_{k}\right\|_{L^{p^{\prime}}} .
\end{aligned}
$$

Passing to the inf, we obtain

$$
\left|\int f h d x\right| \leq\|h\|_{\mathcal{M}^{p, q}}\|f\|_{\mathcal{Z}^{p^{\prime}, q^{\prime}}}
$$


This shows that

$$
\dot{\mathcal{M}}^{p, q} \subset\left(\mathcal{Z}^{p^{\prime}, q^{\prime}}\right)^{*}
$$

To prove the other inclusion take $f \in\left(\mathcal{Z}^{p^{\prime}, q^{\prime}}\right)^{*} \subset L_{l o c}^{p}$. There exists $C_{h}>0$ such that $\forall f \in \mathcal{Z}^{p^{\prime}, q^{\prime}}$

$$
|\langle f, h\rangle| \leq C_{h}\|f\|_{\mathcal{Z}^{p^{\prime}, q^{\prime}}} .
$$

Only remains to prove that $f \in \dot{\mathcal{M}}^{p, q}$. For $x \in \mathbb{R}^{3}$ and $R>0$ we let

$$
f=\bar{h}|h|^{p-2} \chi_{B(x, R)} \in L_{\text {comp }}^{p^{\prime}} .
$$

It follows that

$$
\begin{aligned}
|\langle f, h\rangle| & =\int_{B(x, R)}|h(y)|^{p} d y \leq C_{h} R^{3\left(\frac{1}{q^{\prime}}-\frac{1}{p^{\prime}}\right)}\left\|\bar{h}|h|^{p-2} \chi_{B(x, R)}\right\|_{L^{p^{\prime}}} \\
& \leq C_{h} R^{3\left(\frac{1}{p}-\frac{1}{q}\right)}\left(\int_{B(x, R)}|h(y)|^{p} d y\right)^{\frac{1}{p^{\prime}}} \cdot
\end{aligned}
$$

We infer that

$$
R^{3\left(\frac{1}{p}-\frac{1}{q}\right)}\left(\int_{B(x, R)}|h(y)|^{p} d y\right)^{\frac{1}{p^{\prime}}} \leq C_{h},
$$

and hence $h \in \dot{\mathcal{M}}^{p, q}$. The proof is finished.

The following lemma plays an essential role for the proof of our theorems.

Lemma 2.3 ([14]). Pointwise multiplication is a bounded bilinear operator from $L^{2}\left(\mathbb{R}^{3}\right) \times \dot{H^{r}}\left(\mathbb{R}^{3}\right)$ to $\mathcal{Z}^{p^{\prime}, q^{\prime}}\left(\mathbb{R}^{3}\right)$ for $1 \leq q^{\prime} \leq p^{\prime}<2$ and $\frac{1}{q^{\prime}}=1-\frac{r}{3}$, that is, there exists $C>0$ such that for any $u \in L^{2}\left(\mathbb{R}^{3}\right)$ and $v \in \dot{H}^{r}\left(\mathbb{R}^{3}\right)$,

$$
\|u v\|_{\mathcal{Z}^{p^{\prime}, q^{\prime}}} \leq C\|u\|_{L^{2}\left(\mathbb{R}^{3}\right)}\|v\|_{\dot{H}^{r}\left(\mathbb{R}^{3}\right)} .
$$

For the proof, we refer the reader to Lemarié-Rieusset [14].

\section{Regularity theorem}

In this section we give the regularity criterion by velocity to the Leray type weak solution of the magneto-micropolar fluid equations (1.1). Before turning our attention to regularity issues, we start with some prerequisites for our main result. Let

$$
C_{0, \sigma}^{\infty}\left(\mathbb{R}^{3}\right)=\left\{\varphi \in\left(C_{0}^{\infty}\left(\mathbb{R}^{3}\right)\right): \operatorname{div} \varphi=0\right\} \subseteq\left(C_{0}^{\infty}\left(\mathbb{R}^{3}\right)\right) .
$$

The subspace

$$
L_{\sigma}^{2}\left(\mathbb{R}^{3}\right)=\overline{C_{0, \sigma}^{\infty}\left(\mathbb{R}^{3}\right)}\|\cdot\|_{L^{2}}=\left\{u \in L^{2}\left(\mathbb{R}^{3}\right): \operatorname{div} u=0\right\}
$$


obtained as the closure of $C_{0, \sigma}^{\infty}$ with respect to $L^{2}$-norm $\|\cdot\|_{L^{2}}$. $H_{\sigma}^{r}$ denotes the closure of $C_{0, \sigma}^{\infty}$ with respect to the norm

$$
\|u\|_{H^{r}}=\|u\|_{L^{2}}+\left\|(1-\Delta)^{\frac{r}{2}} u\right\|_{L^{2}}, \text { for } r \geq 0 .
$$

We can now define a notion of weak solution for (1.1) (see e.g. [24]).

Definition 3.1 (weak solutions). Let $\left(u_{0}, b_{0}\right) \in L_{\sigma}^{2}\left(\mathbb{R}^{3}\right), \omega_{0} \in L^{2}\left(\mathbb{R}^{3}\right)$ and $T>0$. A measurable function $(u, \omega, b)$ on $\mathbb{R}^{3} \times(0, T)$ is called a weak solution of $(1.1)$ on $(0, T)$ if

a.

$$
(u, b) \in L^{\infty}\left((0, T) ; L_{\sigma}^{2}\right) \cap L^{2}\left((0, T) ; H_{\sigma}^{1}\right),
$$

and

$$
\omega \in L^{\infty}\left((0, T) ; L^{2}\right) \cap L^{2}\left((0, T) ; H^{1}\right) ;
$$

b. For every $(\phi, \varphi) \in H^{1}\left((0, T) ; H_{\sigma}^{1}\right)$ and $\psi \in H^{1}\left((0, T) ; H^{1}\right)$ with $\phi(T)=$ $\varphi(T)=\psi(T)=0$,

$$
\begin{aligned}
& \int_{0}^{T}\left\{-\left\langle u, \partial_{\tau} \phi\right\rangle+\langle u . \nabla u, \phi\rangle+(\mu+\chi)\langle\nabla u, \nabla \phi\rangle\right\} d \tau \\
& \quad-\int_{0}^{T}\{\langle b . \nabla b, \phi\rangle+\chi\langle\nabla \times \omega, \phi\rangle\}=\left\langle u_{0}, \phi(0)\right\rangle \\
& \int_{0}^{T}\left\{-\left\langle\omega, \partial_{\tau} \varphi\right\rangle+\gamma\langle\nabla \omega, \nabla \varphi\rangle+\kappa\langle\nabla . \omega, \nabla . \varphi\rangle\right\} d \tau \\
& \quad+\int_{0}^{T}\{2 \chi\langle\omega, \varphi\rangle+\langle u . \nabla \omega, \varphi\rangle-\chi\langle\nabla \times u, \varphi\rangle\} d \tau=-\left\langle\omega_{0}, \varphi(0)\right\rangle
\end{aligned}
$$

and

$$
\int_{0}^{T}\left\{-\left\langle b, \partial_{\tau} \psi\right\rangle+\langle u . \nabla b, \psi\rangle+\nu\langle\nabla b, \nabla \psi\rangle-\langle b . \nabla u, \psi\rangle\right\} d \tau=-\left\langle b_{0}, \psi(0)\right\rangle .
$$

Our aim results are to show a new regularity criterion for each of the problems to (1.1).

Theorem 3.1. Let $\left(u_{0}, b_{0}\right) \in \dot{H}_{\sigma}^{1}\left(\mathbb{R}^{3}\right)$ and $\omega_{0} \in \dot{H}^{1}\left(\mathbb{R}^{3}\right)$. Assume that

$$
(u, b) \in C\left((0, T) ; \dot{H}_{\sigma}^{1}\left(\mathbb{R}^{3}\right)\right) \cap C\left((0, T) ; \dot{H}_{\sigma}^{2}\left(\mathbb{R}^{3}\right)\right)
$$

and

$$
\omega \in C\left((0, T) ; \dot{H}^{1}\left(\mathbb{R}^{3}\right)\right) \cap C\left((0, T) ; \dot{H}^{2}\left(\mathbb{R}^{3}\right)\right) .
$$

is a smooth solution to (1.1). If the solution $u$ satisfies one of the following conditions:

$$
u \in L^{\frac{2}{1-r}}\left((0, T) ; \dot{\mathcal{M}}_{p, \frac{3}{r}}\left(\mathbb{R}^{3}\right)\right) \quad \text { for } 0<r<1 \text { and } 2<p<\frac{3}{r}
$$


$(2)$

$$
u \in C\left((0, T) ; \dot{\mathcal{M}}_{p, 3}\left(\mathbb{R}^{3}\right)\right) \quad \text { for } 2<p<\frac{3}{r},
$$

then the solution $(u, \omega, b)$ can be extended smootly beyond $t=T$.

In the reference [19], Rojas-Medar and Boldrini proved the global existence of weak solutions to the Eqs. 1.1 of the magneto-micropolar fluid motion by the Galerkin method. The weak solutions also satisfy the strong energy inequality

$$
\begin{aligned}
& \|(u(t), \omega(t), b(t))\|_{L^{2}}^{2}+2 \mu \int_{\epsilon}^{t}\|\nabla u(s)\|_{L^{2}}^{2} d s \\
& \quad+2 \gamma \int_{\epsilon}^{t}\|\nabla \omega(s)\|_{L^{2}}^{2} d s+2 \nu \int_{\epsilon}^{t}\|\nabla b(s)\|_{L^{2}}^{2} d s+2 \kappa \int_{\epsilon}^{t}\|\nabla u(s)\|_{L^{2}}^{2} d s \\
& \quad+2 \chi \int_{\epsilon}^{t}\|\omega(s)\|_{L^{2}}^{2} d s \leq\left\|u_{0}(\epsilon), \omega_{0}(\epsilon), b_{0}(\epsilon)\right\|_{L^{2}}^{2}
\end{aligned}
$$

for all $0 \leq \epsilon \leq t \leq T$.

Theorem 3.2. Let $\left(u_{0}, b_{0}\right) \in \dot{H}_{\sigma}^{1}\left(\mathbb{R}^{3}\right)$ and $\omega_{0} \in \dot{H}^{1}\left(\mathbb{R}^{3}\right)$. Assume that

$$
(u, b) \in C\left((0, T) ; \dot{H}_{\sigma}^{1}\left(\mathbb{R}^{3}\right)\right) \cap C\left((0, T) ; \dot{H}_{\sigma}^{2}\left(\mathbb{R}^{3}\right)\right)
$$

and

$$
\omega \in C\left((0, T) ; \dot{H}^{1}\left(\mathbb{R}^{3}\right)\right) \cap C\left((0, T) ; \dot{H}^{2}\left(\mathbb{R}^{3}\right)\right) .
$$

is a smooth solution to (1.1). If the solution u satisfies the following condition

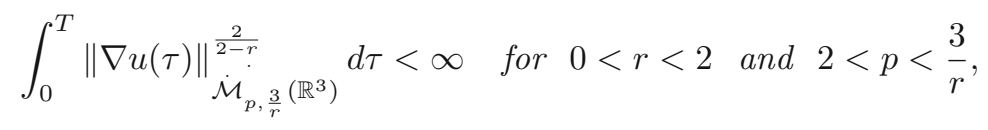

then the solution $(u, \omega, b)$ can be extended smootly beyond $t=T$.

Remark 3.1. Our results improve the results in [24] when $p=q$ and hence $\dot{\mathcal{M}}_{p, p}=L^{p}$. However, it seems to be difficult to prove Theorems 3.1 and 3.2 for $p=2$. Because in the case $p=2$, we have a lack information corresponding to $(2.2)$. There are other difficulties for $p=2$.

\section{Proofs}

We deal with Theorem 3.1 first.

Proof. By differentiating Eqs. 1.1 with respect to $x_{i}$, we take the scalar product with $\partial_{x_{i}} u, \partial_{x_{i}} \omega, \partial_{x_{i}} b$ respectively and integrate over $\mathbb{R}^{3}$. We get after suitable 
integration by parts

$$
\begin{aligned}
\frac{1}{2} \frac{d}{d t} & \left\|\left(\partial_{x_{i}} u, \partial_{x_{i}} \omega, \partial_{x_{i}} b\right)\right\|_{L^{2}}^{2} \\
& +\sum_{j=1}^{3}\left((\mu+\chi)\left\|\partial_{x_{i} x_{j}}^{2} u\right\|_{L^{2}}^{2}+\gamma\left\|\partial_{x_{i} x_{j}}^{2} \omega\right\|_{L^{2}}^{2}+\nu\left\|\partial_{x_{i} x_{j}}^{2} b\right\|_{L^{2}}^{2}\right) \\
& +\kappa\left\|\nabla \cdot \partial_{x_{i}} \omega\right\|_{L^{2}}^{2}+2 \chi\left\|\partial_{x_{i}} \omega\right\|_{L^{2}}^{2} \\
\leq & \left|\left\langle\partial_{x_{i}} u \cdot \nabla u, \partial_{x_{i}} u\right\rangle\right|+\left|\left\langle\partial_{x_{i}} b \cdot \nabla b, \partial_{x_{i}} u\right\rangle\right|+\left|\left\langle\partial_{x_{i}} u \cdot \nabla b, \partial_{x_{i}} b\right\rangle\right| \\
& +\left|\left\langle\partial_{x_{i}} b \cdot \nabla u, \partial_{x_{i}} b\right\rangle\right|+\left|\left\langle\partial_{x_{i}} u \cdot \nabla \omega, \partial_{x_{i}} \omega\right\rangle\right|+2 \chi\left|\left\langle\nabla \times \partial_{x_{i}} u, \partial_{x_{i}} \omega\right\rangle\right| \\
= & \sum_{k=1}^{6} A_{k}
\end{aligned}
$$

for $t \in(0, T)$, where we used

$$
\begin{aligned}
& \left\langle\nabla \times \partial_{x_{i}} u, \partial_{x_{i}} \omega\right\rangle=\left\langle\nabla \times \partial_{x_{i}} \omega, \partial_{x_{i}} u\right\rangle, \\
& \left\langle b . \nabla \partial_{x_{i}} b ., \partial_{x_{i}} u\right\rangle+\left\langle b . \nabla \partial_{x_{i}} u, \partial_{x_{i}} b\right\rangle=0
\end{aligned}
$$

and

$$
\left\langle u . \nabla \partial_{x_{i}} u ., \partial_{x_{i}} u\right\rangle=\left\langle u . \nabla \partial_{x_{i}} b, \partial_{x_{i}} b\right\rangle=0 .
$$

The first term $A_{1}$ is dominated by the Hölder and Young inequalities such that

$$
\begin{aligned}
A_{1} & =\left|-\int_{\mathbb{R}^{3}} u \cdot \nabla u \cdot \partial_{x_{i}^{2}}^{2} u d x\right| \\
& \leq\left\|D^{2} u \cdot \nabla u\right\|_{Z_{p^{\prime}, \frac{3}{3-r}}}\|u\|_{\dot{\mathcal{M}}_{p, \frac{3}{r}}} \\
& \leq\left\|D^{2} u\right\|_{L^{2}}\|u\|_{\dot{\mathcal{M}}_{p, \frac{3}{r}}}\|\nabla u\|_{\dot{H}^{r}}^{r} \\
& \leq\left\|D^{2} u\right\|_{L^{2}}\|u\|_{\dot{\mathcal{M}}_{p, \frac{3}{r}}}\|\nabla u\|_{\dot{H}^{1}}^{r}\|\nabla u\|_{L^{2}}^{1-r} \\
& \leq\left\|D^{2} u\right\|_{L^{2}}^{1+r}\left(\|u\|_{\dot{\mathcal{M}}_{p, \frac{3}{r}}^{\frac{2}{1-r}}}^{1+\nabla u \|_{L^{2}}^{2}}\right)^{\frac{1-r}{2}} \\
& \leq \frac{\chi}{12}\left\|D^{2} u\right\|_{L^{2}}^{2}+C_{r}\|u\|_{\dot{\mathcal{M}}_{p, \frac{3}{r}}^{1-r}}^{\frac{2}{1-r}}\|\nabla u\|_{L^{2}}^{2},
\end{aligned}
$$

where we used the following inequality for $0 \leq r \leq 1$

$$
\|w\|_{\dot{H}^{r}}=\left\||\xi|^{r} \widehat{w}\right\|_{L^{2}}=\left(\int_{\mathbb{R}^{3}}|\xi|^{2 r}|\widehat{w}|^{2 r}|\widehat{w}|^{2-2 r} d \xi\right) \leq\|w\|_{L^{2}}^{1-r}\|\nabla w\|_{L^{2}}^{r} .
$$

Similarly, we can estimate $A_{2}$ as

$$
\begin{aligned}
A_{2} & =\left|-\int_{\mathbb{R}^{3}} u \cdot \nabla b \cdot \partial_{x_{i}^{2}}^{2} b d x\right| \\
& \leq\left\|D^{2} b \cdot \nabla b\right\|_{Z_{p^{\prime}, \frac{3}{3-r}}}\|u\|_{\dot{\mathcal{M}}_{p, \frac{3}{r}}} \\
& \leq \frac{\nu}{12}\left\|D^{2} b\right\|_{L^{2}}^{2}+C_{r}\|u\|_{\dot{\mathcal{M}}_{p, \frac{3}{r}}^{1-r}}^{\frac{2}{1-r}}\|\nabla b\|_{L^{2}}^{2} .
\end{aligned}
$$


In the same way, for $A_{3}, A_{4}$ and $A_{5}$, it also can be dominated as

$$
A_{3}, A_{4} \leq \frac{\nu}{18}\left\|D^{2} b\right\|_{L^{2}}^{2}+C_{r}\|u\|_{\mathcal{M}_{p, \frac{3}{r}}}^{\frac{2}{1-r}}\|\nabla b\|_{L^{2}}^{2}
$$

and

$$
A_{5} \leq \frac{\gamma}{6}\left\|D^{2} \omega\right\|_{L^{2}}^{2}+C_{r}\|u\|_{\mathcal{\mathcal { M }}_{p, \frac{3}{r}}}^{\frac{2}{1-r}}\|\nabla \omega\|_{L^{2}}^{2} .
$$

The last term $A_{6}$ can be dealt with a similar manner. By the Hölder and Young inequalities we have

$$
A_{6} \leq \frac{\chi}{2}\left\|\nabla \times \partial_{x_{i}} u\right\|_{L^{2}}^{2}+2 \chi\|\nabla \omega\|_{L^{2}}^{2}
$$

From the above inequalities and summing over $i$ with $1 \leq i \leq 3$, we have

$$
\begin{aligned}
& \frac{d}{d t}\|(\nabla u, \nabla \omega, \nabla b)\|_{L^{2}}^{2}+\left(2 \mu+\frac{1}{2} \chi\right)\left\|D^{2} u\right\|_{L^{2}}^{2} \\
& \quad+\gamma\left\|D^{2} \omega\right\|_{L^{2}}^{2}+\nu\left\|D^{2} b\right\|_{L^{2}}^{2}+2 \kappa\|\nabla \operatorname{div} \omega\|_{L^{2}}^{2} \\
& \leq C_{r}\|u\|_{\mathcal{M}_{p, \frac{3}{r}}^{\frac{2}{1-r}}}^{2}\|(\nabla u, \nabla \omega, \nabla b)\|_{L^{2}}^{2} .
\end{aligned}
$$

Due to Gronwall' s inequality, it follows from (4.1) that

$$
\begin{aligned}
& \sup _{0 \leq t \leq T}\|(\nabla u(t), \nabla \omega(t), \nabla b(t))\|_{L^{2}}^{2} \\
& \quad \leq\left\|\nabla u_{0}, \nabla \omega_{0}, \nabla b_{0}\right\|_{L^{2}}^{2} \exp \left(C \int_{0}^{t}\|u(s)\|_{\dot{\mathcal{M}}_{p, \frac{3}{r}}}^{\frac{2}{2-r}} d s\right) .
\end{aligned}
$$

as $0<r<1$.

For $r=1$, we follow the proof of Zhou-Gala [28] (see also [11,16]). To this end, we need the following fact: for $u \in C\left((0, T) ; \dot{\mathcal{M}}_{p, 3}\left(\mathbb{R}^{3}\right)\right)$ and arbitrary positive $\epsilon>0$, there exists a decomposition

$$
u=u_{1}+u_{2}
$$

such that for all $T>0$,

$$
\left\|u_{1}\right\|_{u \in C\left((0, T) ; \dot{\mathcal{M}}_{p, 3}\left(\mathbb{R}^{3}\right)\right)} \leq \epsilon, \quad \sup _{(t, x) \in(0, T) \times \mathbb{R}^{3}}\left|u_{2}(t, x)\right|<K(\epsilon) .
$$

One can simply choose

$$
u_{2}(x, t)= \begin{cases}u(x, t) & \text { for }|u(t, x)|<K, \\ 0 & \text { for }|u(t, x)| \geq K,\end{cases}
$$


where $K$ is sufficiently large. We first estimate $A_{1}$ :

$$
\begin{aligned}
A_{1} & \leq \int_{\mathbb{R}^{3}}|u|\left|D^{2} u\right||\nabla u| d x \\
& \leq\left\|D^{2} u \cdot \nabla u\right\|_{Z_{p^{\prime}, \frac{3}{2}}}\left\|u_{1}\right\|_{\dot{\mathcal{M}}_{p, 3}}+\left\|D^{2} u\right\|_{L^{2}}\left\|u_{2} \cdot \nabla u\right\|_{L^{2}} \\
& \leq\left\|D^{2} u\right\|_{L^{2}}\left(\left\|u_{1}\right\|_{\dot{\mathcal{M}}_{p, 3}}\|\nabla u\|_{\dot{H}^{1}}+K(\epsilon)\|\nabla u\|_{L^{2}}\right) \\
& \leq\left\|D^{2} u\right\|_{L^{2}}\left(\epsilon\left\|D^{2} u\right\|_{L^{2}}+K(\epsilon)\|\nabla u\|_{L^{2}}\right) \\
& \leq \frac{\chi}{12}\left\|D^{2} u\right\|_{L^{2}}^{2}+K(\epsilon)\|\nabla u\|_{L^{2}}^{2} .
\end{aligned}
$$

Similarly, for $A_{2}, A_{3}$ and $A_{4}$, we obtain

$$
A_{2}, A_{3}, A_{4} \leq \frac{\nu}{18}\left\|D^{2} b\right\|_{L^{2}}^{2}+K(\epsilon)\|\nabla b\|_{L^{2}}^{2}
$$

and

$$
A_{5} \leq \frac{\gamma}{6}\left\|D^{2} \omega\right\|_{L^{2}}^{2}+K(\epsilon)\|\nabla \omega\|_{L^{2}}^{2} .
$$

Combining the above estimates and summing up $i$, it follows

$$
\begin{aligned}
& \frac{d}{d t}\|(\nabla u, \nabla \omega, \nabla b)\|_{L^{2}}^{2}+\left(2 \mu+\frac{1}{2} \chi\right)\left\|D^{2} u\right\|_{L^{2}}^{2} \\
& \quad+\gamma\left\|D^{2} \omega\right\|_{L^{2}}^{2}+\nu\left\|D^{2} b\right\|_{L^{2}}^{2}+2 \kappa\|\nabla \operatorname{div} \omega\|_{L^{2}}^{2} \\
& \quad \leq K(\epsilon)\|(\nabla u, \nabla \omega, \nabla b)\|_{L^{2}}^{2} .
\end{aligned}
$$

Hence, by using Gronwall' s inequality, we have

$$
\sup _{0 \leq t \leq T}\|(\nabla u(t), \nabla \omega(t), \nabla b(t))\|_{L^{2}}^{2} \leq\left\|\nabla u_{0}, \nabla \omega_{0}, \nabla b_{0}\right\|_{L^{2}}^{2} \exp (C K(\epsilon) T) .
$$

This completes the proof of Theorem 3.1.

Proof of Theorem 3.2.

Proof. Similarly as in the proof of Theorem 3.1, we differentiate the equations (1.1) with respect to $x_{i}$, then multiply the resulting equations by $\partial_{x_{i}} u$, $\partial_{x_{i}} \omega, \partial_{x_{i}} b$ respectively, we obtain after suitable integration by parts

$$
\begin{aligned}
\frac{1}{2} \frac{d}{d t} & \left\|\left(\partial_{x_{i}} u, \partial_{x_{i}} \omega, \partial_{x_{i}} b\right)\right\|_{L^{2}}^{2} \\
& +\sum_{j=1}^{3}\left((\mu+\chi)\left\|\partial_{x_{i} x_{j}}^{2} u\right\|_{L^{2}}^{2}+\gamma\left\|\partial_{x_{i} x_{j}}^{2} \omega\right\|_{L^{2}}^{2}+\nu\left\|\partial_{x_{i} x_{j}}^{2} b\right\|_{L^{2}}^{2}\right) \\
& +\kappa\left\|\nabla \cdot \partial_{x_{i}} \omega\right\|_{L^{2}}^{2}+2 \chi\left\|\partial_{x_{i}} \omega\right\|_{L^{2}}^{2} \\
\leq & \left|\left\langle\partial_{x_{i}} u \cdot \nabla u, \partial_{x_{i}} u\right\rangle\right|+\left|\left\langle\partial_{x_{i}} b . \nabla b, \partial_{x_{i}} u\right\rangle\right|+\left|\left\langle\partial_{x_{i}} u . \nabla b, \partial_{x_{i}} b\right\rangle\right| \\
& +\left|\left\langle\partial_{x_{i}} b \cdot \nabla u, \partial_{x_{i}} b\right\rangle\right|+\left|\left\langle\partial_{x_{i}} u \cdot \nabla \omega, \partial_{x_{i}} \omega\right\rangle\right|+2 \chi\left|\left\langle\nabla \times \partial_{x_{i}} u, \partial_{x_{i}} \omega\right\rangle\right| \\
= & \sum_{k=1}^{6} B_{k}
\end{aligned}
$$


for $t \in(0, T)$. Then Young's inequality implies that

$$
\begin{aligned}
& B_{1}=\left|\int_{\mathbb{R}^{3}} \partial_{x_{i}} u \cdot \nabla u \cdot \partial_{x_{i}} u d x\right| \\
& \leq\|D u \cdot D u\|_{Z_{p^{\prime}, \frac{3}{3-r}}}\|\nabla u\|_{\mathcal{\mathcal { M }}_{p, \frac{3}{r}}} \\
& \leq\|D u\|_{L^{2}}\|\nabla u\|_{\mathcal{M}_{p, \frac{3}{r}}}\|D u\|_{\dot{H}^{r}} \\
& \leq\|D u\|_{L^{2}}\|\nabla u\|_{\mathcal{M}_{p, \frac{3}{r}}}\left\|D^{2} u\right\|_{L^{2}}^{r}\|D u\|_{L^{2}}^{1-r} \\
& \leq\left(\left\|D^{2} u\right\|_{L^{2}}^{2}\right)^{\frac{r}{2}}\left(\|\nabla u\|_{\mathcal{M}_{p, \frac{3}{r}}^{2-r}}^{\frac{2}{2-r}}\|D u\|_{L^{2}}^{2}\right)^{\frac{2-r}{2}} \\
& \leq \frac{\chi}{12}\left\|D^{2} u\right\|_{L^{2}}^{2}+C_{r}\|\nabla u\|_{\mathcal{\mathcal { M }}_{p, \frac{3}{r}}}^{\frac{2}{2-r}}\|D u\|_{L^{2}}^{2} .
\end{aligned}
$$

Similarly, for $B_{2}-B_{5}$, we have

$$
B_{2}, B_{3}, B_{4} \leq \frac{\nu}{18}\left\|D^{2} b\right\|_{L^{2}}^{2}+C_{r}\|\nabla u\|_{\mathcal{M}_{p, \frac{3}{r}}^{2-r}}^{\frac{2}{2-r}}\|D b\|_{L^{2}}^{2}
$$

and

$$
B_{5} \leq \frac{\gamma}{6}\left\|D^{2} \omega\right\|_{L^{2}}^{2}+C_{r}\|\nabla u\|_{\mathcal{\mathcal { M }}_{p, \frac{3}{r}}}^{\frac{2}{2-r}}\|D \omega\|_{L^{2}}^{2} .
$$

Combining the above estimates and summing over $i$ with $1 \leq i \leq 3$, we obtain

$$
\begin{aligned}
& \frac{d}{d t}\|(\nabla u, \nabla \omega, \nabla b)\|_{L^{2}}^{2}+\left(2 \mu+\frac{1}{2} \chi\right)\left\|D^{2} u\right\|_{L^{2}}^{2} \\
& \quad+\gamma\left\|D^{2} \omega\right\|_{L^{2}}^{2}+\nu\left\|D^{2} b\right\|_{L^{2}}^{2}+2 \kappa\|\nabla \operatorname{div} \omega\|_{L^{2}}^{2} \\
& \leq C_{r}\|\nabla u\|_{\dot{X}_{r}}^{\frac{2}{2-r}}\|(\nabla u, \nabla \omega, \nabla b)\|_{L^{2}}^{2} .
\end{aligned}
$$

Due to Gronwall' s inequality, it follows from (4.3) that

$$
\begin{aligned}
& \sup _{0 \leq t \leq T}\|(\nabla u(t), \nabla \omega(t), \nabla b(t))\|_{L^{2}}^{2} \\
& \quad \leq\left\|\nabla u_{0}, \nabla \omega_{0}, \nabla b_{0}\right\|_{L^{2}}^{2} \exp \left(C \int_{0}^{t}\|\nabla u(s)\|_{\mathcal{\mathcal { M }}_{p, \frac{3}{r}}^{2-r}}^{\frac{2}{2-r}} d s\right) .
\end{aligned}
$$

By the standard arguments of continuation of local solutions, we conclude that the solutions $(u(x, t), \omega(x, t), b(x, t))$ can be extendted to $\left(0, T^{\prime}\right)$ for some $T^{\prime}>T$, provided that $\nabla u$ satisfies

$$
\int_{0}^{T}\|\nabla u(\tau)\|_{\dot{\mathcal{M}}_{p, \frac{3}{r}}^{2-r}\left(\mathbb{R}^{3}\right)}^{\frac{2}{2}} d \tau<\infty \text { for all } 0<r<2 .
$$

This completes the proof of Theorem 3.2.

Remark 4.1. But this method fails if $p=2$. To avoid the difficulty, we have to modify the definition of the critical Morrey-Campanato space $\dot{\mathcal{M}}_{2, \frac{3}{r}}$. For the details, the reader is referred to [6]. 


\section{Acknowledgments}

The author would like to thank the referee for his careful reading of the work and his many helpful suggestions. Thanks are also due to Professor Yong Zhou for his useful comments and encouragement.

\section{References}

[1] Caflish, R.E., Klapper, I., Steel, G.: Remarks on singularities, dimension and energy dissipation for ideal hydrodynamics and MHD. Comm. Math. Phys. 184, 443-455 (1997)

[2] Cannone, M., Miao, C.X., Prioux, N., Yuan, B.Q.: The Cauchy Problem for the Magneto-hydrodynamic System. Self-similar Solutions of Nonlinear PDE, vol. 74, pp. 59-93. Banach Center Publications, Institute of Mathematics, Polish Academy of Sciences, Warszawa (2006)

[3] Duvant, G., Lions, J.L.: Inéquations en thermoélasticite et magnetohydrodynamique. Arch. Ration. Mech. Anal. 46, 241-279 (1972)

[4] Eringen, A.C.: Theory of micropolar fluids. J. Math. Mech. 16, 1-18 (1996)

[5] Ferreira, L.C.F., Villamizar-Roa, E.J.: On the existence and stability of solutions for the micropolar fluids in exterior domains. Math. Meth. Appl. Sci. 30, 11851208 (2007)

[6] Gala, S., Yuan, B.: Remarks on the regularity of weak solutions to magnetomicropolar fluid equations (2009)

[7] Galdi, G.P., Rionero, S.: A note on the existence and uniqueness of solutions of the micropolar fluid equations. Internat. J. Eng. Sci. 15, 105-108 (1997)

[8] He, C., Wang, Y.: On the regularity criteria for weak solutions to the magnetohydrodynamic equations. J. Differ. Equ. 238, 1-17 (2007)

[9] He, C., Xin, Z.P.: On the regularity of weak solutions to the magnetohydrodynamic equations. J. Differ. Eq. 213, 235-254 (2005)

[10] Kato, T.: Strong $L^{p}$ solutions of the Navier-Stokes equations in Morrey spaces. Bol. Soc. Bras. Mat. 22, 127-155 (1992)

[11] Kozono, H.: Weak solutions of the Navier-Stokes equations with test functions in the weak- $L^{n}$ space. Tohoku Math. J. 53, 55-79 (2001)

[12] Kozono, H., Yamazaki, M.: Semilinear Heat equations and the Navier-Stokes equation with distributions in new function spaces as initial data. Comm. P. D. E. 19, 959-1014 (1994)

[13] Ladyzhenskaya, O.: The Mathematical Theory of Viscous Incompressible Flows. Gordon and Breach, New York (1969)

[14] Lemarié-Rieusset, P.G.: Recent Developments in the Navier-Stokes Problem. Chapman \& Hall/CRC, London (2002) 
[15] Lions, P.L.: Mathematical Topics in Fluid Mechanics. Oxford University Press Inc., New York (1996)

[16] Lions, P.L., Masmoudi, N.: Uniqueness of mild solutions of the Navier-Stokes system in $L^{N}$. Comm. P. D. E. 26, 2211-2226 (2001)

[17] Ortega-Torres, E.E., Rojas-Medar, M.A.: Magneto-micropolar fluid motion: global existence of strong solutions. Abstr. Appl. Anal. 4, 109-125 (1999)

[18] Rojas-Medar, M.A.: Magneto-micropolar fluid motion: existence and uniqueness of strong solutions. Math. Nachr. 188, 301-319 (1997)

[19] Rojas-Medar, M.A., Boldrini, J.L.: Magneto-micropolar fluid motion: existence of weak solutions. Rev. Mat. Complut. 11, 443-460 (1998)

[20] Sermange, M., Temam, R.: Some mathematical questions related to the MHD equations. Comm. Pure Appl. Math. 36, 635-664 (1983)

[21] Taylor, M.E.: Analysis on Morrey spaces and applications to Navier-Stokes and other evolution equations. Comm. P. D. E. 17, 1407-1456 (1992)

[22] Villamizar-Roa, E.J., Rodríguez-Bellido, M.A.: Global existence and exponential stability for the micropolar fluid system. Z. Angew. Math. Phys. 59, 790809 (2008)

[23] Yamaguchi, N.: Existence of global strong solution to the micropolar fluid system in a bounded domain. Math. Meth. Appl. Sci. 28, 1507-1526 (2005)

[24] Yuan, B.Q.: Regularity of weak solutions to magneto-micropolar fluid equations. Acta Math. Sci. (in press)

[25] Zhou, Y.: Remarks on regularities for the 3D MHD equations. Discrete Continuous Dyn. Syst. 12, 881-886 (2005)

[26] Zhou, Y.: Regularity criteria for the 3D MHD equations in terms of the pressure. Intl. J. Non-Linear Mech. 41, 1174-1180 (2006)

[27] Zhou, Y.: Regularity criteria for the generalized viscous MHD equations. Ann. Inst. H. Poincaré Anal. Non Linéaire 24, 491-505 (2007)

[28] Zhou, Y., Gala, S.: Regularity criteria for the solutions to the 3D MHD equations in the multiplier space. ZAMP (in press)

S. Gala

Department of Mathematics, University of Mostaganem,

Box 227, Mostaganem 27000, Algeria

e-mail: sadek.gala@gmail.com

Received: 01 March 2009.

Accepted: 30 October 2009. 